Evaluation of the use of saturated salt solutions for the museum relative humidity control

\author{
Enas Abo El enen Amin1,*, Mohammed Tharwat Mohammed 2 \\ 1 Associate Professor of Textiles Conservation, Conservation Dept, Faculty of Fine Arts, Minia \\ University, Minia, Egypt \\ 2 Lecturer of Metal Conservation, Conservation Dept, Faculty of Fine Arts, Minia University, \\ Minia, Egypt
}

\title{
Evaluation of the use of saturated salt solutions for the museum relative humidity control
}

\begin{abstract}
RH that is too high, too low or that fluctuates widely damages archaeological collections. Saturated salt solutions (Magnesium chloride, Sodium bromide, Potassium carbonate) have been used to obtain an equilibrium relationship of relative humidity and temperature. Over a temperature range $30^{\circ} \mathrm{C} \pm 2^{\circ} \mathrm{C}$. The experiments described in this research have the intention of creating known environmental conditions in containers where the linen textiles and metal samples are to be tested. The aim is to evaluate of saturated salt solutions to reach humidity to equilibrium state over a three-weeks and their effect on experimental samples. The scanning electron microscopy (SEM) and electron dispersive X-ray spectroscopy (EDX and EDS) analysis were used to detect the presence of salt ions deposition in samples. The mechanical characterize of textile samples were tested by tensile strength and elongation test.
\end{abstract}

Keywords: Saturated salt solutions; Magnesium chloride; Sodium bromide; Potassium carbonate; metal samples; EDX; EDS; textile samples.

\section{INTRODUCTION}

The deterioration and preservation of materials depends on two things: the nature of the material and the environment surrounding the material (Lampropoulos et al., 2007). Organic materials, such as wood, paper, cotton, linen, wool, silk, parchment, leather, fur, feathers, ivory, bone and horn, also retain moisture. These materials are hygroscopic. They can and will absorb or give off moisture until they reach a state of equilibrium with the air that surrounds them. When the surrounding air is damp, the materials will absorb some of the moisture from the air: they may swell, cockle, warp, change shape and/or lose strength (Slade, 2003). Dampness in the presence of oxygen can also cause mould and fungal growth on organic materials (Bacci et al., 2008; Georgianna et al., 2003; Henderson, 2007). Fragile objects with a layered structure, such as veneered furniture, behave in two ways: they can expand and contract at different rates lengthwise and widthwise. This has the same effect as if the object was made of different materials (Cassar and Hutching, 2000). All metal objects corrode due to interaction with its surroundings. Corrosion is a never-ending phenomenon, the rate depending upon the nature of the metal and the severity of the reacting agencies. This is also a fact that corrosion is much more rapid and severe in humid climate, because most chemical reactions take place with the help of moisture (Agrawal, 1987; Brown et al., 2001). The object loss of the shape and loss of its original surface as the object breaks up (Rimmer and Wang, 2010). A stable and safe microenvironment able to enclose objects and enhance security is the appropriate solutions and showcases comply with those requirements (Romano et al., 2013). The aim is to stable relative 
humidity when controlling temperature and humidity as stable as possible (Scottish Museums Council Fact Sheet, 2003). A stable level of RH, even if it approximates target levels, is preferable to maintaining exact desired levels for only part of the time (Trustees of the Royal Air Force Museum, 2008). The common methods of controlling the humidity accurately use either a humidity generator or the equilibration of a closed space with a chemical system which produces the desired equilibrium vapor pressure. Humidity generators tend to be expensive and complex and only one humidity level is obtained each time. Among the chemical systems used for this purpose are saturated salt solutions (Greenspan, 1977; Jialu, 2011; Wexler and Hasegawa, 1954). The aqueous salt solutions were found to be cheap enough to get. Since a given saturated salt solution provides only one relative humidity level at any desired temperature, a different relative humidity level must be achieved by selecting another appropriate salt (Dostal, 2013; Hyland and Hurley, 1983). The time for salts to reach equilibrium is within an hour in room temperature with mechanical convection (Jialu, 2011). By providing excess solute, the solution will remain saturated even in the presence of modest sources or sinks. Where the solute is a solid in the pure phase, it is easy to determine that there is indeed saturation (Greenspan, 1977). Disadvantages of this system are that a different salt is needed for each humidity, a salt for a specific humidity may not be available, many of the salts are corrosive and the equilibrium relative humidity RH formed over many solutions varies widely with temperature (Forney and Brandl, 1992). For that the humidifying process in this research lasts for a weeks to assure that the whole system reaches the humidity equilibrium.

This paper presents many samples and tests have to be done before obtaining reliable results. The experiments envisaged in the scope of this research aim to have constant temperature and relative humidity throughout the whole testing period (Quincot et al., 2011).

\section{EXPERIMENTAL AND SAMPLES DESCRIPTION}

The aim of the experimental program is to evaluate of saturated salt solutions to reach humidity to equilibrium state over a three-weeks and their effect on experimental samples.

\subsection{Preparation of samples}

2.1.1. Unbleached plain linen textiles samples

Fabric was further purified in the laboratory by scouring by boiling in water for 1 hour and then boiled in a solution containing $2 \mathrm{~g} / \mathrm{L}$ of sodium carbonate for $1 \frac{1 / 2}{2}$ hour in order to remove natural wax and other impurities. Samples were thoroughly washed with tap water and dried at ambient conditions as Elgaoudi et al., (2011). Finally, the linen samples were cut into 15/3 cm strips.

\subsubsection{The metal samples (Bronze)}

Bronze alloy (copper about $74 \%$, zinc about $23 \%$, and traces about $3 \%$ ) is prepared, the dimensions of slide is $1.5 \mathrm{~cm} \times 1 \mathrm{~cm} \times 1 \mathrm{~mm}$ as a thickness, and in its edge is a small hole for hanging, and make sure before putting the samples cleaned with acetone and complete drying with cleaning soft cotton cloth.

\section{2. $\quad$ The thermal aging}

The thermal aging aimed to the production of artificially aged model of linen fabrics similar as possible to the archaeological ones, taking into account that the thermal degradation increases with increasing the temperature degree and the duration of aging (Elgaoudi et al., 2011). The linen samples were artificially thermally aged at $140{ }^{\circ} \mathrm{C}$ in precision forced convection oven for $72 \mathrm{~h}$ according to Feller (1994). He presented that heating paper for $72 \mathrm{~h}$ ( 3 days) at $100^{\circ} \mathrm{C}$ is equivalent to about 25 
years of ageing under normal condition ageing. He also showed that heating paper for $36 \mathrm{~h}$ at $140^{\circ} \mathrm{C}$ is equivalent to about 100 years of ageing under normal conditions.

\subsection{Containers}

Three transparent containers were used with the dimensions:

24 width $\times 19$ length $\times 17$ heights (Figure 1$)$.

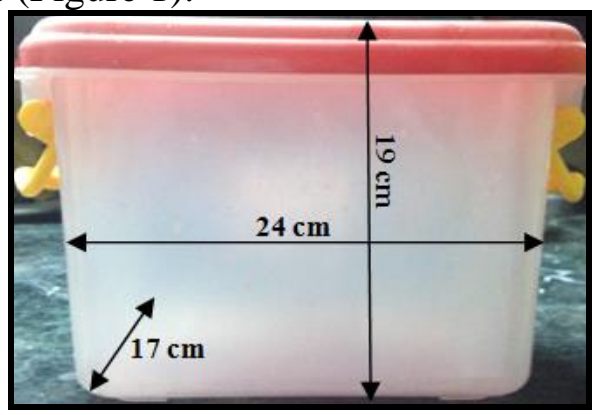

Figure 1. The containers dimensions

The textiles and Bronze samples were hanged with thermal thread on the above of the containers, the humidity sensors were hanged on the side of the containers for measuring humidity (Figure 2), (Samples do not contact with the solution), allowing to relate the salt solution efficiency in view of the targeted relative humidity in the container, and its stability along time.

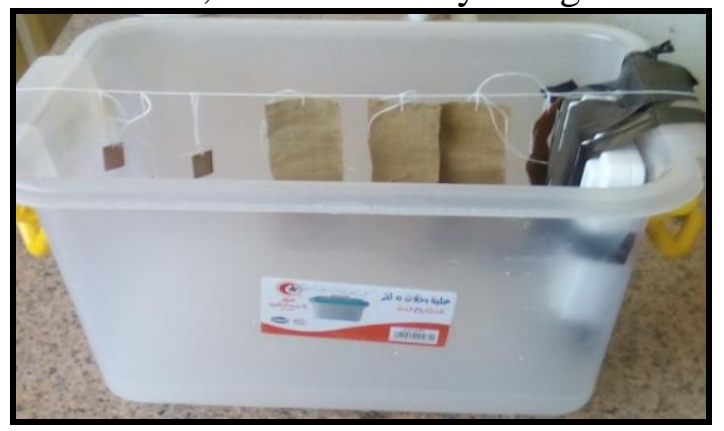

Figure 2. Hanging the textiles and bronze samples by thermal thread

\subsection{Preparation of saturated salt solutions}

Three salts were used (Magnesium chloride, Sodium bromide, Potassium carbonate). To prepare a saturate salt solution, the indicated salt is added to warm (about $40{ }^{\circ} \mathrm{C}$ ) distilled water with stirring until no more salt dissolves. Additional salt is added to ensure an excess of the saturating salt. Table 1 provides approximate compositions for saturated solutions. The saturated solution is then cooled to ambient temperature and allowed to set for at least 24 hours before use. All salt crystals should be covered by the solution according to (Blaine, 2004; Jowitt and Wagstaffe, 1989). Each salt solution was placed in a separate container (Table.1).

Table 1.The percent of salts which used (by grams) to water ( by milliliter).

\begin{tabular}{lcc}
\hline \multicolumn{1}{c}{ Salt } & Salt $(\mathrm{g})$ & Water $(\mathrm{mL})$ \\
\hline Magnesium chloride & 200 & 25 \\
Sodium bromide & 200 & 80 \\
Potassium carbonate & 200 & 80 \\
\hline
\end{tabular}

\subsection{Controlling the temperature}

The temperature was controlled by placing all the containers in the growth chamber (incubation) and adjusting them for three weeks at $30^{\circ} \mathrm{C} \pm 2^{\circ} \mathrm{C}$ (Figure 3 ). 


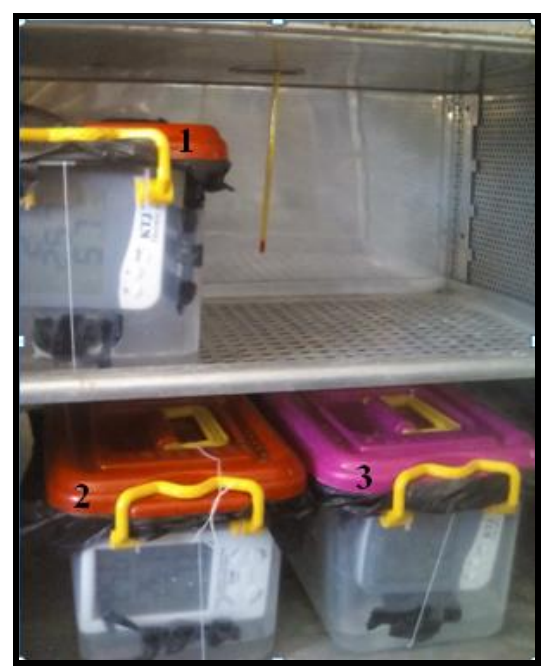

Figure 3. The growth chamber contains containers for three weeks at $30^{\circ} \mathrm{C} \pm 2^{\circ} \mathrm{C}$

\section{ANALYTICAL TECHNIQUES}

\subsection{RH measurements for the containers with different salts}

The objective is to monitor the behavior of the salts along the time, to compare the readings of each sensor for each salt solution, and to check the influence of the salt solution on the RH measurements. Check the stability of the temperature along the time at $30^{\circ} \mathrm{C}$.

\subsection{Textile samples}

3.2.1. Scanning Electron Microscope (SEM) equipped with EDX analysis

The investigation was carried in the Egyptian Mineral Resources Authority, Central Laboratories Sector. The textiles samples were investigated by using Scanning Electron Microscope (Using SEM Model Quanta 250 FEG (Field Emission Gun)) to study any damage was happened on these fibers, or any salts was deposited in samples. The Scanning Electron Microscope for samples attached with EDX Unit (Energy Dispersive X-ray Analyses, and K550X sputter coater England, with accelerating voltage $30 \mathrm{~K}$.V., magnification14x up to 1000000 and resolution for Gun.1n).To analysis the different elements in the samples.

\subsubsection{Mechanical behavior}

The mechanical parameters such as tensile strength and elongation of linen samples were determined. three samples per treatment set were tested and the breaking load averaged for each sample (Ahmed, 2013), by using tensile testing machine before and after the saturated salt solution effect using En ISO 13934-1;1999 Maximum Force \& Elongation - strip Method under Load Range $100 \mathrm{~N}$, Extension Range $20 \mathrm{~mm}$, Gauge Length $100 \mathrm{~mm}$, Speed $100 \mathrm{~mm} / \mathrm{min}$ and Preload $2.0 \mathrm{~N}$. Three samples were used for each test and statistical data were calculated. (The test was carried in the National Institute of Standards in Cairo).

\subsection{Bronze samples}

Scanning Electron Microscope (SEM) equipped with EDS analysis:

The investigation was carried in Central Laboratory for Microanalysis, Minia University. The bronze samples were investigated by using Scanning Electron Microscope (SEM), Model JSM-IT200, version 
2 , field of view $55.65 \mu \mathrm{M} 41.74 \mu \mathrm{M}$, with image size 1280 x 960, Then use Energy Dispersive X-Ray Spectroscopy (EDS) analysis

\section{RESULTS AND DISCUSSION}

4.1. $\quad$ RH measurements for the containers with different salts

The readings of the humidity sensors in each container are shown in (table 2) (Figure 4a,b,c).

Table 2. Read of the humidity measurements at three weeks in each container.

\begin{tabular}{|c|c|c|c|c|c|c|c|c|c|c|c|c|c|c|c|c|c|c|c|c|c|}
\hline \multirow{3}{*}{$\begin{array}{c}\text { The } \\
\text { Salts } \\
\mathrm{MgCl}_{2}\end{array}$} & \multirow{2}{*}{\multicolumn{7}{|c|}{ The First Week }} & \multirow{2}{*}{\multicolumn{7}{|c|}{ The second Week }} & \multirow{2}{*}{\multicolumn{7}{|c|}{ The third Week }} \\
\hline & & & & & & & & & & & & & & & & & & & & & \\
\hline & 32 & 32 & 32 & 32 & 32 & 32 & 32 & 32 & 32 & 35 & 38 & 41 & 41 & 42 & 42 & 45 & 48 & 51 & 51 & 51 & 51 \\
\hline $\mathrm{NaBr}$ & 56 & 56 & 56 & 56 & 56 & 56 & 56 & 56 & 58 & 61 & 61 & 65 & 67 & 68 & 68 & 69 & 70 & 70 & 70 & 70 & 70 \\
\hline $\mathrm{K}_{2} \mathrm{CO}_{3}$ & 42 & 43 & 43 & 43 & 43 & 43 & 43 & 43 & 43 & 46 & 46 & 50 & 52 & 52 & 53 & 55 & 56 & 58 & 61 & 61 & 61 \\
\hline
\end{tabular}
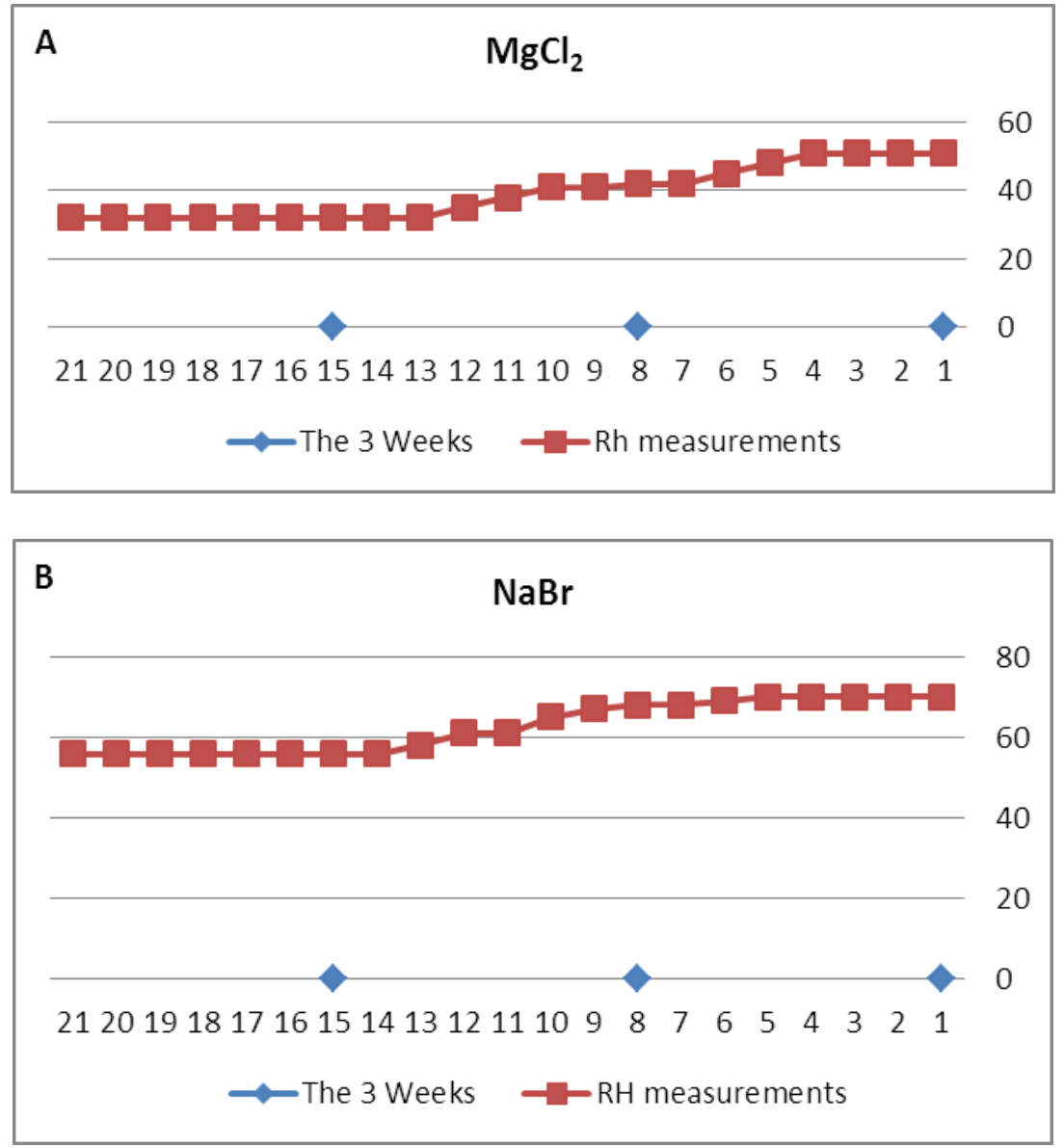


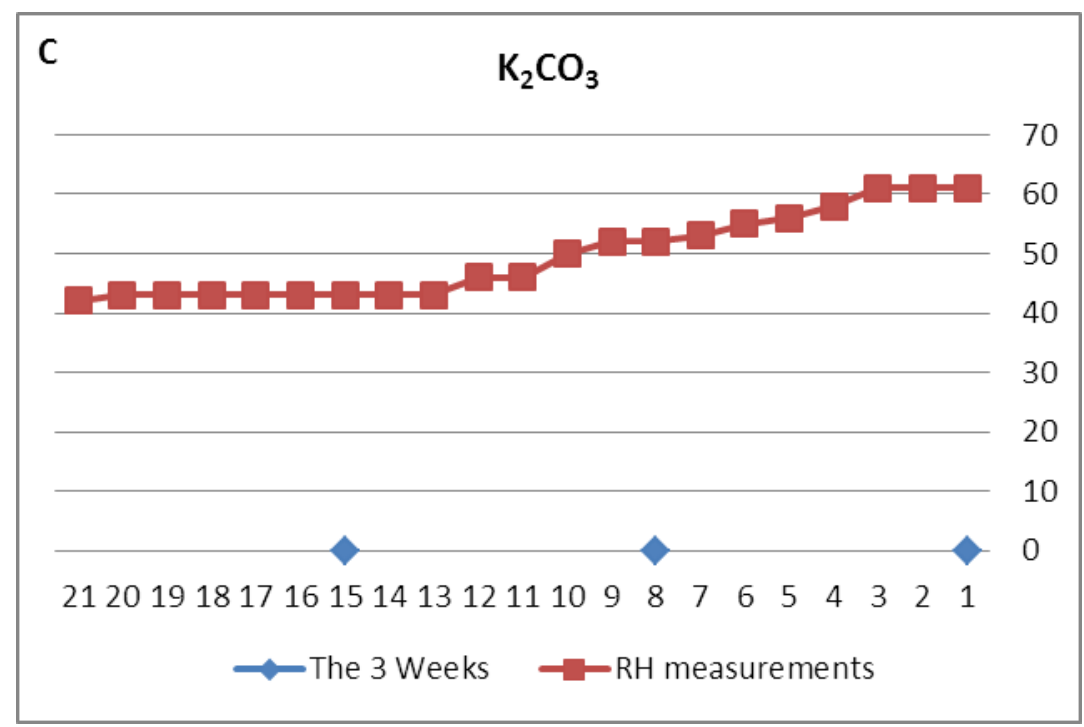

Figure 4a,b,c. Graph for RH readings of each salt

The RH measurements for the three salts at the stability temperature during the first week of the experiment showed constant stability. The readings for all salts started to rise gradually during the second and third weeks. The readings reached stability again at the end of the third week. There is a rise in $\mathrm{RH}$ measurements of sodium bromide salt to a great extent up to $70 \%$ and this degree of moisture is not acceptable and gives risky condition in conservation of the archaeological materials if it is used above one week. Potassium carbonate salt showed an acceptable increase in $\mathrm{RH}$ measurements up to the second week in a degree that can be used with many archaeological materials for two weeks. Magnesium chloride salt in spite of the high humidity measurements in the second and third week, but at the end of the experiment was taken suitable measurements for the conservation of different types of archaeological materials for three weeks.

\subsection{Textile samples \\ 4.2.1. SEM photos}

The SEM photos showed deposition of salts on the surface of linen samples with $\mathrm{NaBr}$ and $\mathrm{K}_{2} \mathrm{CO}_{3}$ salts compared with the standard sample which showed free of salts but there is some dust (Figure $5 \mathrm{a}, \mathrm{b}, \mathrm{c}$ ). There is no deposition of salts with $\mathrm{MgCl}_{2}$ salt (Figure 5c). There are cracks and damages on the fibers due to the aging in the all photos.

A

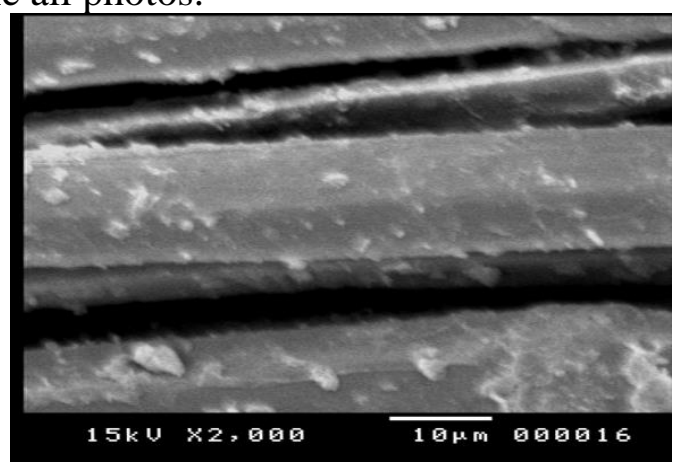


B
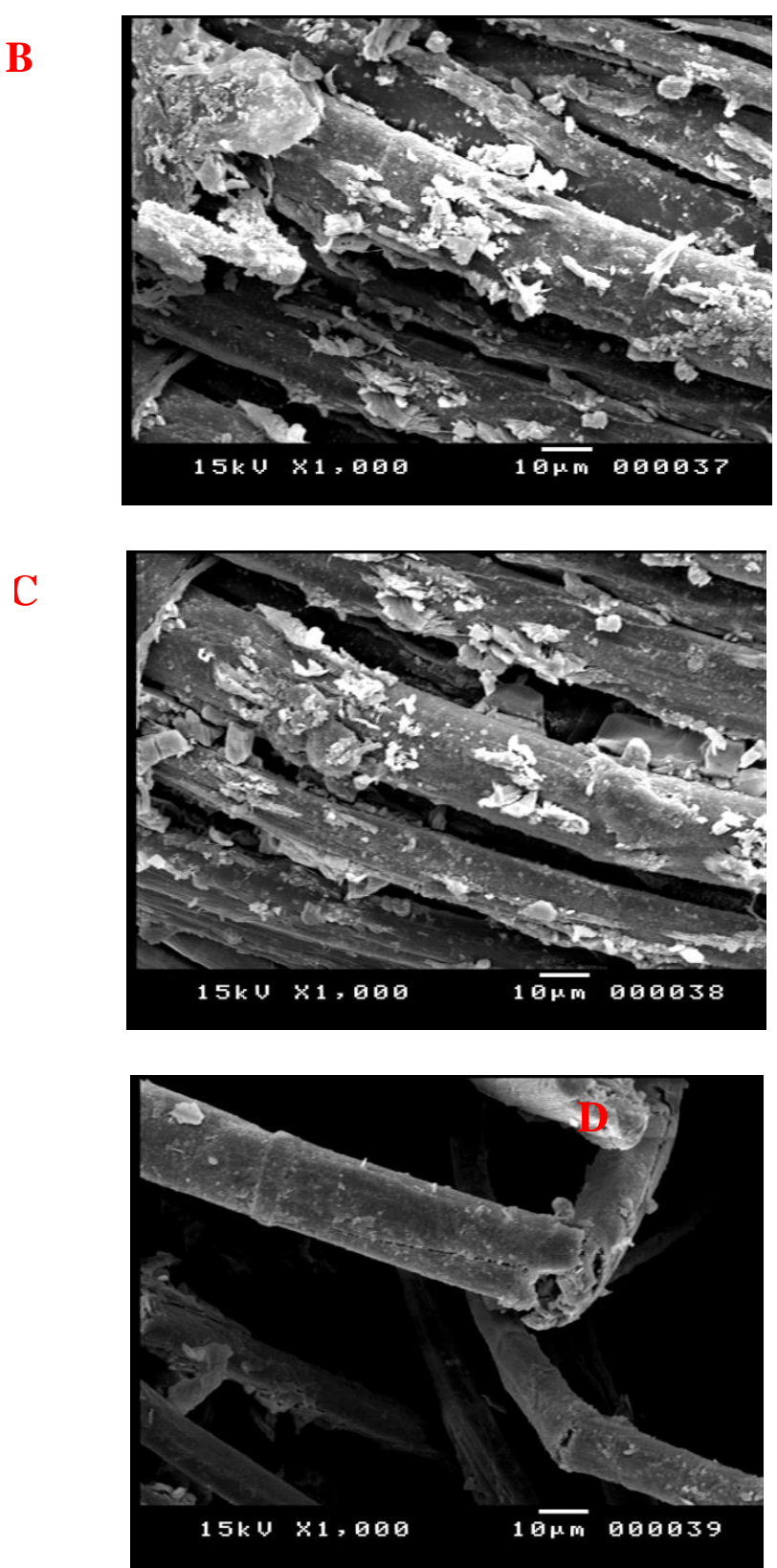

Figure 5a. The standard sample, b.The SEM photo showed that there is deposition of salts on the surface of linen sample with $\mathrm{NaBr}$ salt, c. There is deposition of salts on the surface of sample with $\mathrm{K}_{2} \mathrm{CO}_{3}$ salt, $\mathrm{d}$. There is no deposition of the salts with $\mathrm{MgCl}_{2}$ salt

\subsubsection{EDX analysis}

Different linen surface samples were investigated by using EDX in order to study the deposited of the Salts elements on the samples surface. The results showed that there are a small percent of $\mathrm{Br}$ and $\mathrm{Na}$ elements on the linen surface sample which is hanging with the $\mathrm{NaBr}$ salt solution and that's evidence of salt deposition within the sample pores (Figure 6). The results showed that there is $\mathrm{K}$ element on the linen surface sample which is hanging with the $\mathrm{K}_{2} \mathrm{Co}_{3}$ salt solution (Figure 7). The results also showed that is no salts elements were present on the linen surface sample which was hanged with the $\mathrm{MgCl}_{2}$ salt solution (Figure 8). 


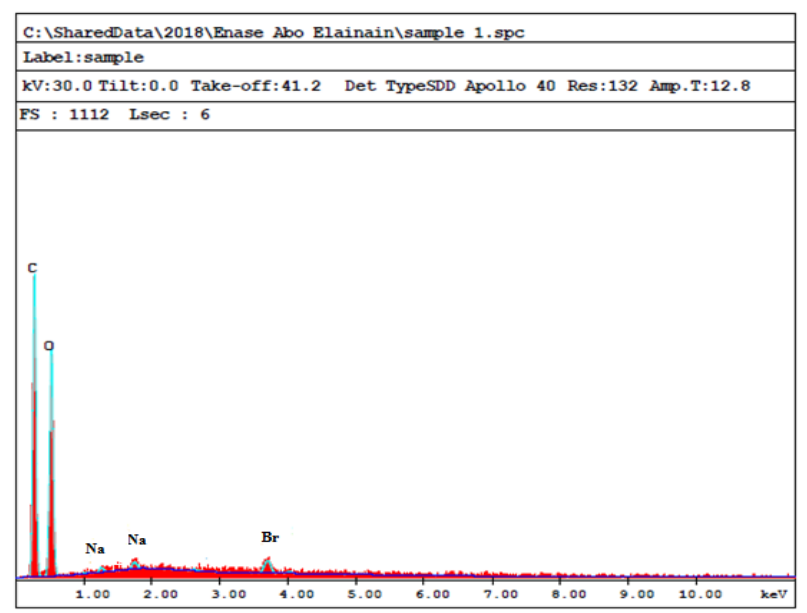

Figure 6. EDX analysis showed that there are $\mathrm{Br}$ and $\mathrm{Na}$ elements on the linen surface

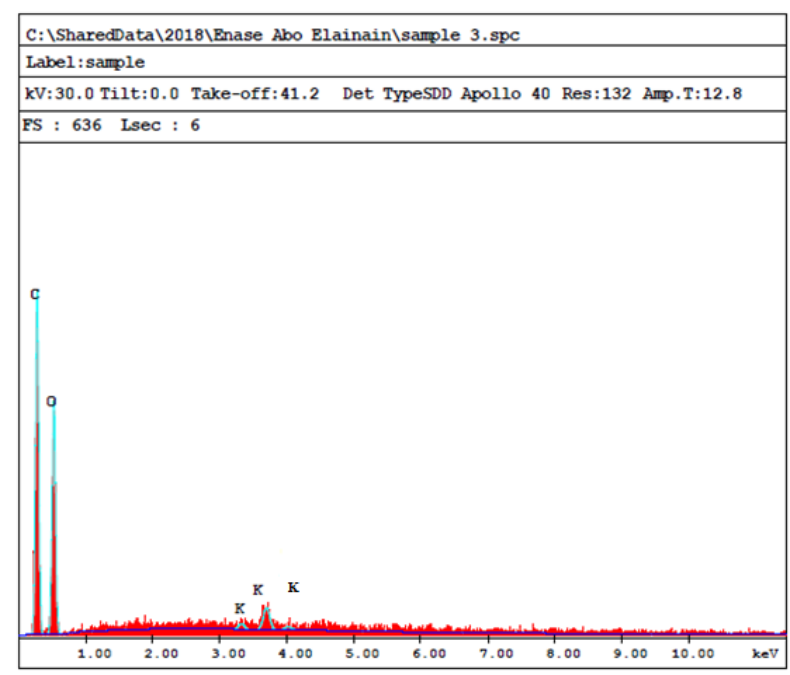

Figure 7. EDX analysis showed that there are K elements on the linen sample

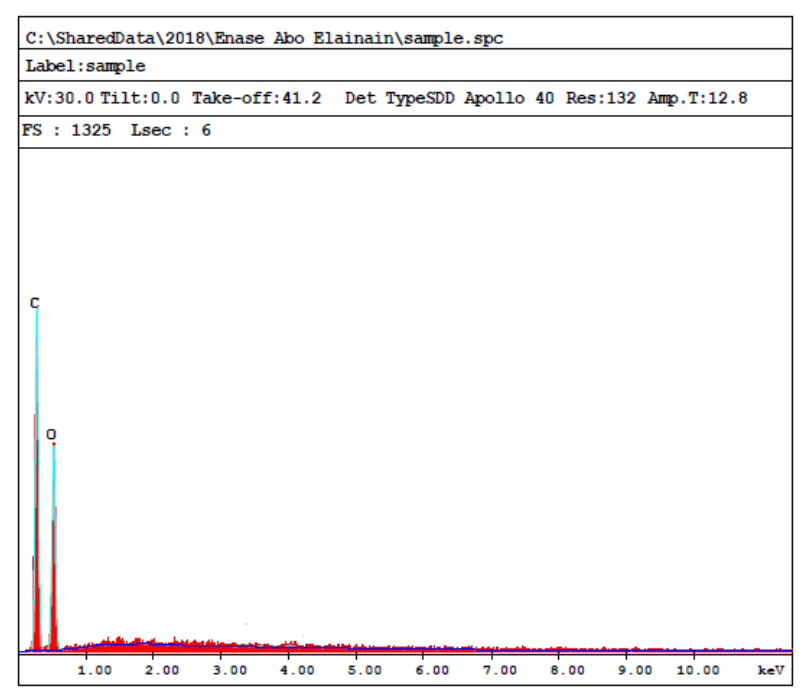


Figure 8. EDX analysis showed that there are no elements were present on the linen surface sample which hanging with the $\mathrm{MgCl}_{2}$ salt solution

\subsubsection{Mechanical behavior}

Changes in the mechanical properties could be attributed to increasing the RH levels. The linen samples which were hanged with the $\mathrm{MgCl}_{2}$ salt solution show a slight decrease in elongation and tensile strength, compared to standard samples (The samples after ageing without placing it with salt solutions). The samples which were hanged with the $\mathrm{K}_{2} \mathrm{CO}_{3}$ salt solution show a little decrease in elongation and tensile strength, compared to standard samples. The samples which were hanged with the $\mathrm{NaBr}$ salt solution show a big decrease in elongation and tensile strength as shown in (Table 3) and (Figure 9a,b) .

Table 3. Effect of salt solutions on Mechanical parameters such as Tensile strength and Elongation of linen samples.

\begin{tabular}{|c|c|c|c|}
\hline \multirow{6}{*}{ 를 } & \multicolumn{3}{|c|}{ Mechanical parameters } \\
\hline & Samples & Tensile strength (kgf) & Elongation (mm) \\
\hline & Standard & 164.6 & 26.74 \\
\hline & $\mathrm{MgCl}_{2}$ & 164.0 & 26.71 \\
\hline & NaBr & 163.03 & 24.41 \\
\hline & $\mathrm{K}_{2} \mathrm{CO}_{3}$ & 163. 72 & 25.63 \\
\hline
\end{tabular}

A

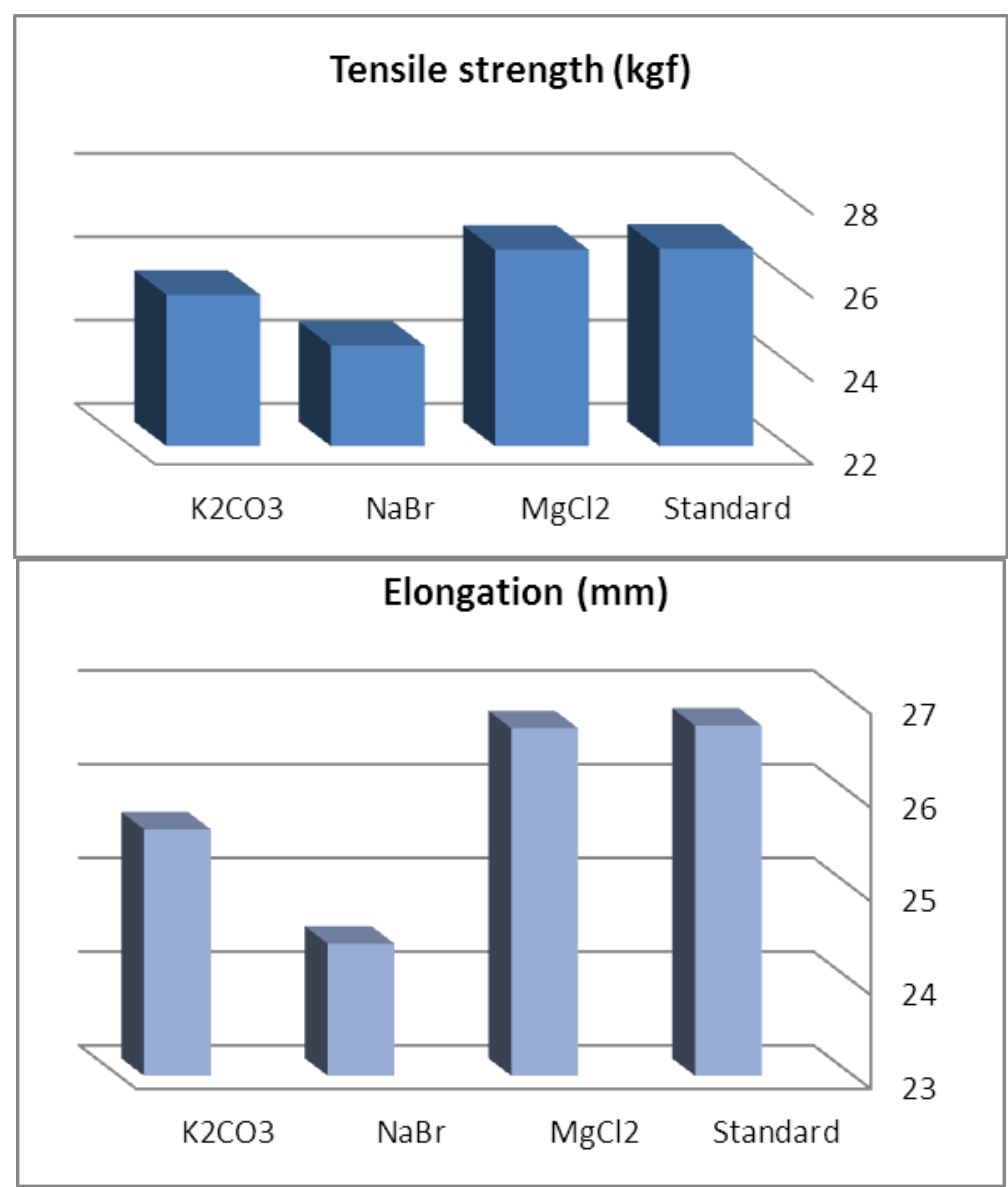


Figure 9. Show the effect of Salt solutions on Mechanical parameters such as Tensile strength (A) and Elongation (B) of linen samples

\subsection{Bronze samples}

4.3.1. Digital Microscope

The results of the microscopy examination (Figure 10) in the sample (I) showed the significant nonimpact of potassium carbonate salts $\left(\mathrm{K}_{2} \mathrm{Co}_{3}\right)$. In the sample (II) the appearance of different colors of rust to the effect of salts of Magnesium Chloride $\left(\mathrm{MgCl}_{2}\right)$ tends to reddish but without affecting the surface of bronze. In the sample (III) the Sodium Bromide salts (NaBr) significantly affected the bronze from where the rust colors and the surface of the metal, where protrusions and calcinations of the salts were spread as a green color on the surface of the sample.

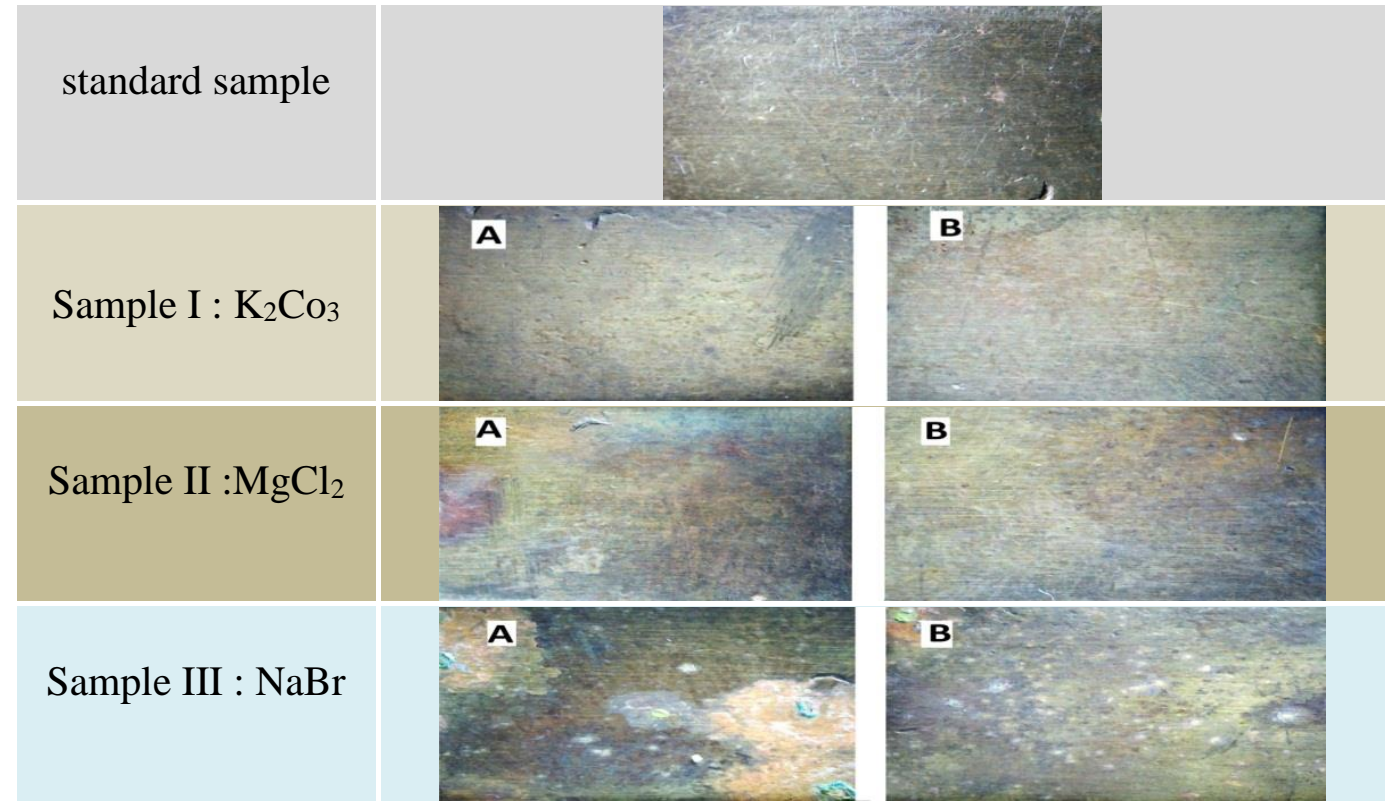

Figure 10. Digital microscope image (200x) standard sample, Sample I : $\mathrm{K}_{2} \mathrm{Co}_{3}$ with sides (A,B), Sample II : $\mathrm{MgCl}_{2}$ with sides (A,B), and Sample III : $\mathrm{NaBr}$ with sides (A,B)

\subsubsection{SEM photos}

In the sample of Potassium Carbonate salt $\left(\mathrm{K}_{2} \mathrm{Co}_{3}\right)$, the bronze sample showed in $1500 \mathrm{X}$ increase in surface salinity (Figure 11). In the sample of the salts of the Magnesium Chloride $\left(\mathrm{MgCl}_{2}\right)$, with magnification power $(2300 \mathrm{X})$ showed crystallization of the salts with medium size and appearance of protrusions in the surface of bronze as a start of rust steps (Figure 12). In the third sample of Sodium Bromide salt $(\mathrm{NaBr})$, the salinity spread and its effect were apparent in the image (A), even with a simple magnification power. (140X) The rust layers were clearly visible (Figure 13a). In the image (B) with a magnification power (1000X) showed the edge of bronze sample was in the begging step of separation and have clearly crack as a reason of salt effect (Figure 13b). 


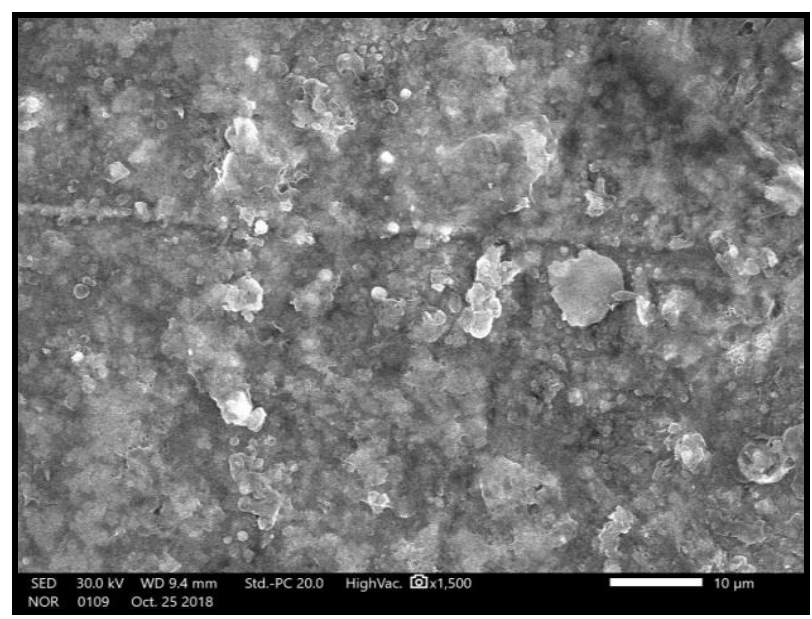

Figure 11. SEM image for bronze sample affected by salt $\mathrm{K}_{2} \mathrm{Co}_{3}$ with magnification power (1500x) showed salinity of the surface with no corrosion

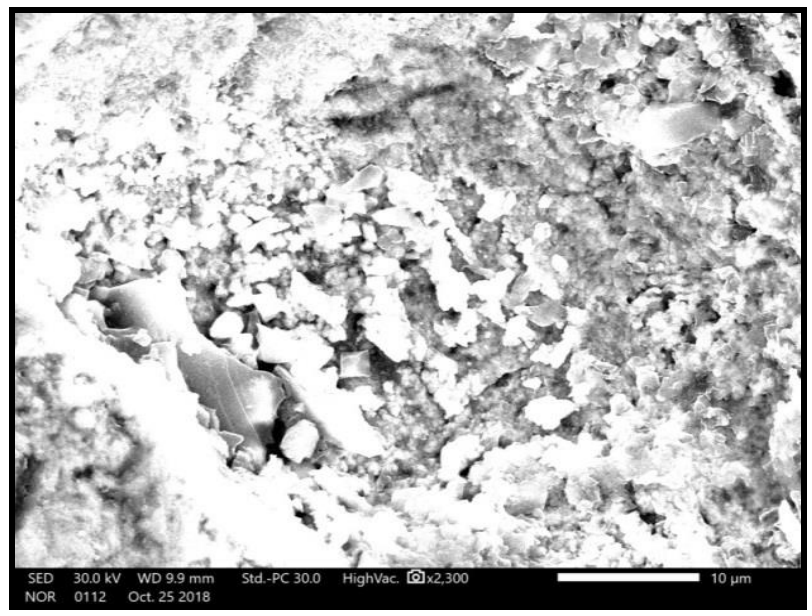

Figure 12. SEM image for bronze sample affected by salt $\mathrm{MgCl}_{2}$ with magnification power (2300x) showed crystallization of the salt with appearance of protrusions in the surface

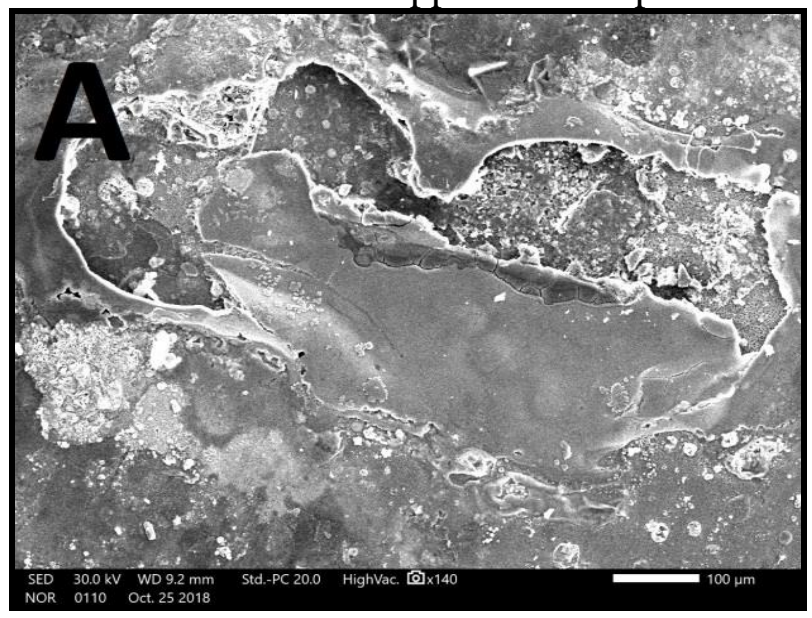




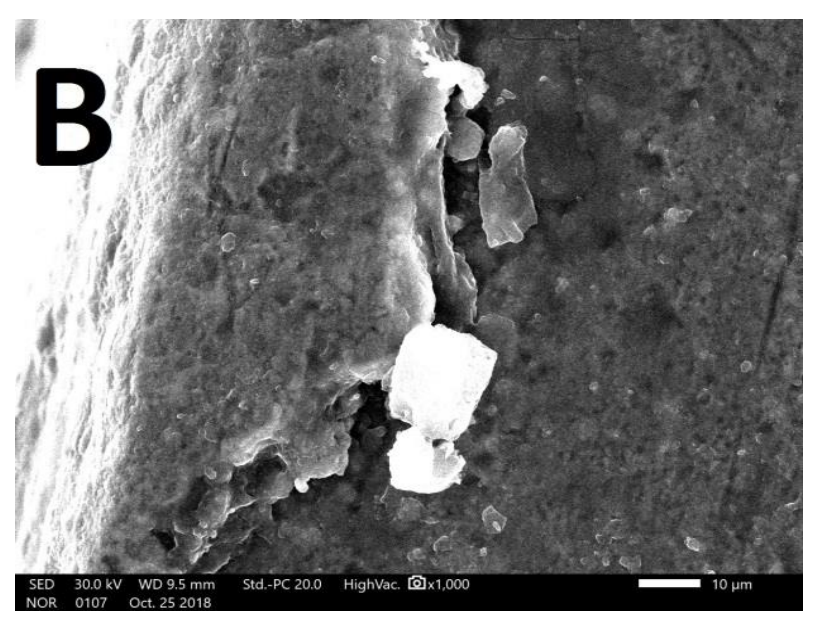

Figure 13a. SEM image for bronze sample affected by salt $\mathrm{NaBr}$ with magnification power (140x) showed the salinity and rust spread. B. SEM image for bronze sample affected by salt $\mathrm{NaBr}$ with magnification power (1000x) in the edge of the sample begging step of separation and have clearly

\subsubsection{EDS analysis}

crack

In sample No.(1) Potassium Carbonate salt $\left(\mathrm{K}_{2} \mathrm{Co}_{3}\right)$, the result of analyze showed elements of copper $(\mathrm{Cu})$ with percent $70.26 \%$ and zinc $(\mathrm{Zn})$ with percent $4.36 \%$ as a main component of bronze alloy, the elements of Carbon (C), and Oxygen (O) with percent $14.08 \%, 8.62 \%$ respectively as a component of the Carbonate $\left(\mathrm{CO}_{3}\right)$. Then the elements of Potassium $(\mathrm{K})$ with percent $1.73 \%$, Niobium $(\mathrm{Nb})$ element with percent $0.88 \%$ and Calcium $(\mathrm{Ca})$ element with percent $0.07 \%$ as a traces (Figure 14, 15) (Table 4). And the mapping with chart showed the spread of elements in the sample by coloring each element with a deferent color (Figure 16).

So we can to conclude to the Potassium Carbonate not effective as a damage salt on bronze surface except the low mass of zinc than the normal percent may be refer to appearance of an oxidation compounds, and appearance of Niobium element that's used in the holder of the instrument's analyze, Potassium and Calcium elements may be as an air impurity.

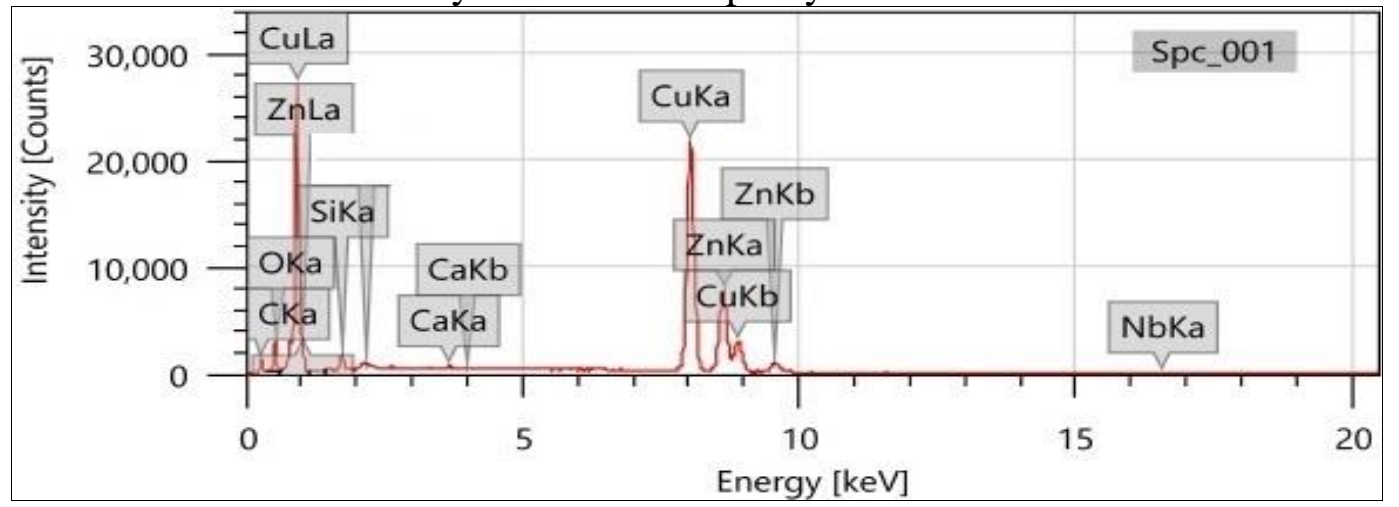

Figure 14. EDS analyses evident the elements in bronze sample affected by $\mathrm{K}_{2} \mathrm{Co}_{3}$ salt

Table 4. Percents of mass and atom of each element in bronze sample affected with $\mathrm{K}_{2} \mathrm{Co}_{3}$ salt 


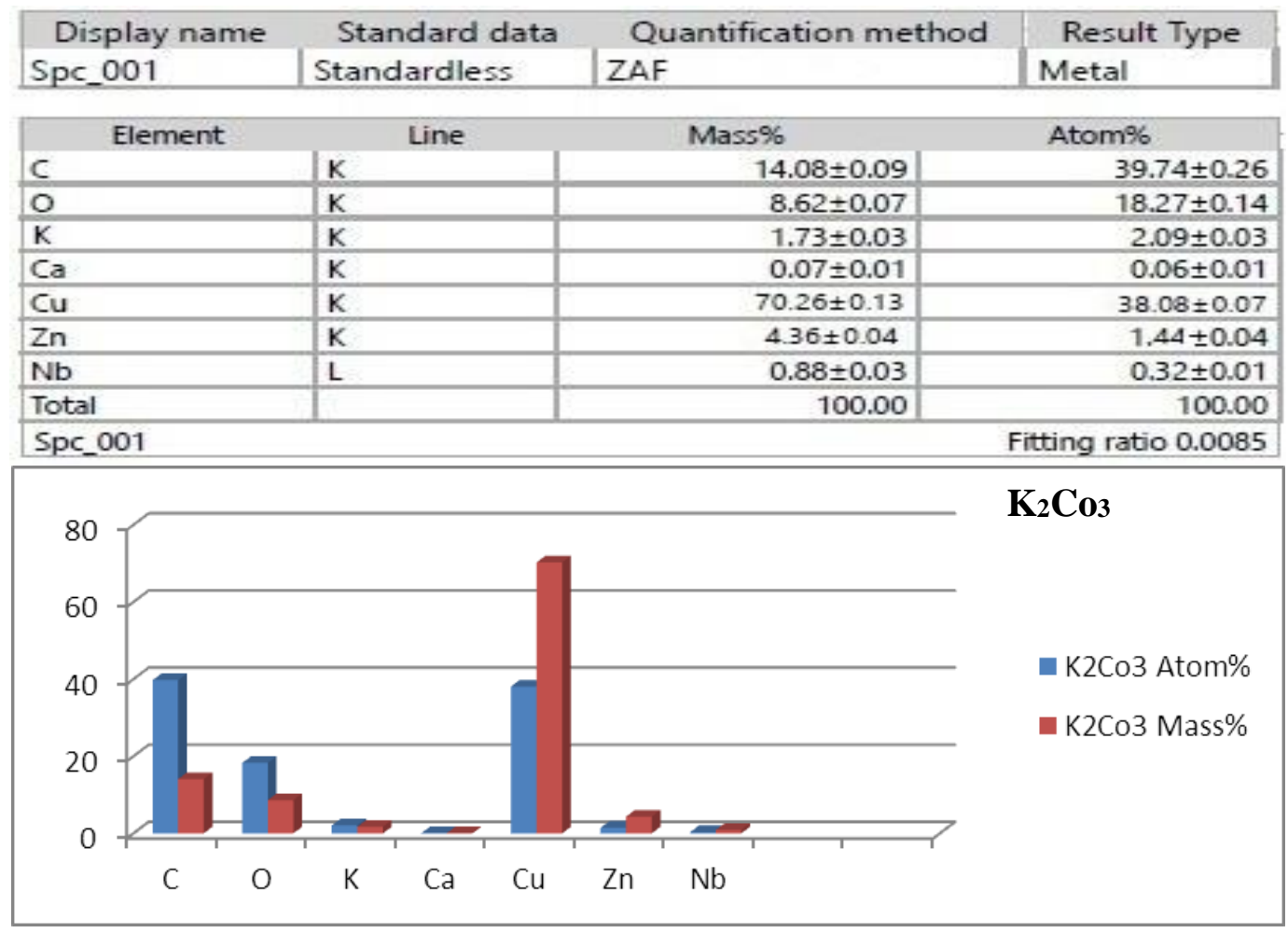

Figure 15. Graph comparing the elements in bronze sample affected by $\mathrm{K}_{2} \mathrm{Co}_{3}$ salt
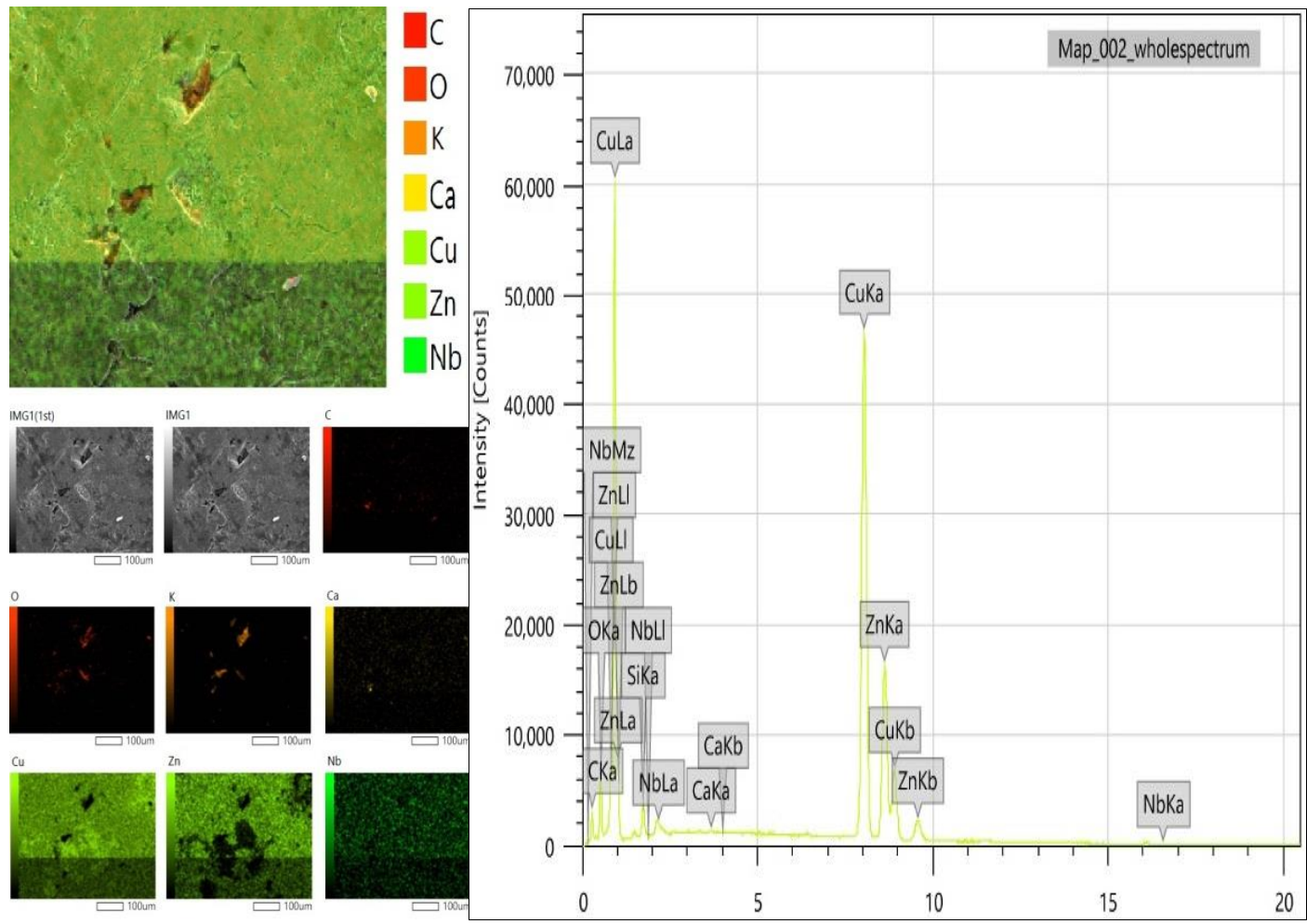
Figure 16. Map and chart show the spread of elements in the sample by coloring each element with a deferent color in bronze sample affected by $\mathrm{K}_{2} \mathrm{Co}_{3}$ salt

In sample No.(2) Magnesium Chloride $\left(\mathrm{MgCl}_{2}\right)$, the result of analyze showed elements of copper $(\mathrm{Cu})$ with percent $53.91 \%$ and Zinc $(\mathrm{Zn})$ with percent $20.44 \%$ as a main component of bronze alloy. The elements of Carbon (C) and Oxygen (O) with percent $12.78 \%, 9.75 \%$ respectively. The Gold ( $\mathrm{Au})$ element with percent $1.16 \%$, then the Magnesium $(\mathrm{Mg})$ element with percent $1.03 \%$, and Chloride $(\mathrm{Cl})$ element with percent $0.25 \%$. Finally the Sulfur (S) element with percent $0.44 \%$ and Calcium (Ca) element with percent $0.24 \%$, as a traces (Figure 17, 18) (Table 5). And the mapping with chart showed the spread of elements in the sample (Figure 19).

So we can to conclude to the Magnesium Chloride salt effect on the percent of copper in bronze alloy significantly, and not effective on zinc element, this makes the salt has a negative effect on the sample. The gold element is found because using it as a way of preparing the sample by coating. So we can say it happen an oxidation and carbonization processes formed a change in the percent of an element especially copper as a basic composite element which in turn gave rise to forms of rust as in SEM photos.

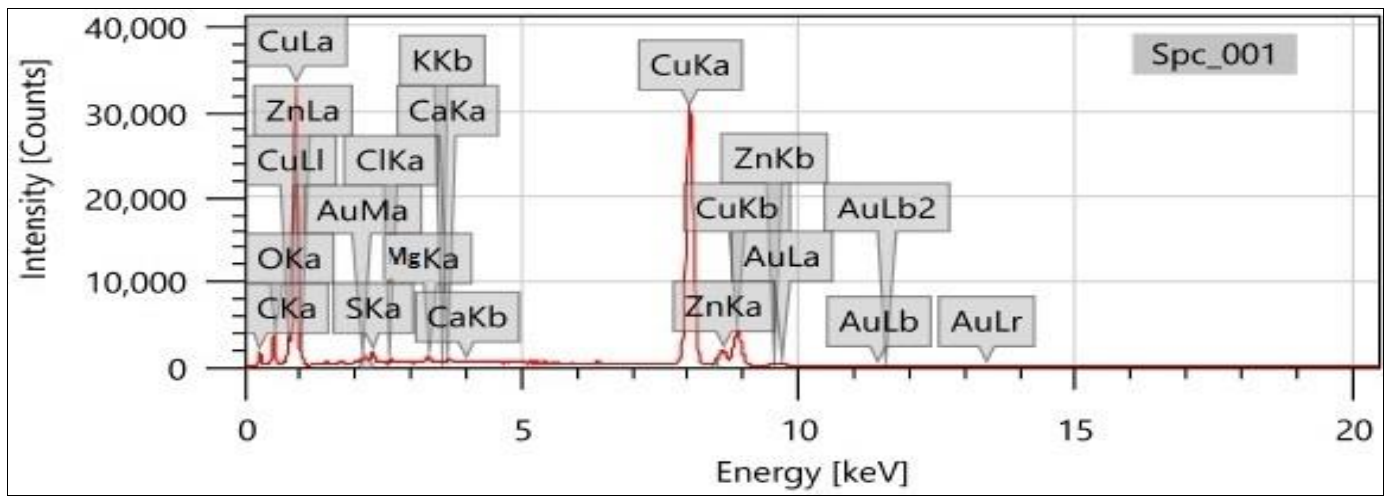

Figure 17. EDS analyses evident the elements in bronze sample affected by $\mathrm{MgCl}_{2}$ salt

Table 5. Percents of mass and atom of each element in bronze sample affected by $\mathrm{MgCl}_{2}$ salt

\begin{tabular}{|c|c|c|c|}
\hline Display name & Standard da & Quantification method & Result Type \\
\hline Spc_00 & Standardless & ZAF & Metal \\
\hline \multicolumn{2}{|l|}{ Element } & Mass $\%$ & Atom $\%$ \\
\hline c & K & $12.78 \pm 0.08$ & $36.63 \pm 0.23$ \\
\hline 0 & $\mathrm{~K}$ & $9.75 \pm 0.07$ & $20.99 \pm 0.14$ \\
\hline Mg & K & $1.03 \pm 0.01$ & $1.11 \pm 0.02$ \\
\hline $\mathrm{Cl}$ & K & $0.25 \pm 0.01$ & $0.24=0.01$ \\
\hline s & K & $0.44 \pm 0.01$ & $0.39=0.01$ \\
\hline $\mathrm{Ca}$ & K & $0.24 \pm 0.01$ & $0.21 \pm 0.01$ \\
\hline $\mathrm{Cu}$ & K & $53.91 \pm 0.12$ & $28.78 \pm 0.07$ \\
\hline $\mathrm{Zn}$ & $\mathrm{k}$ & $20.44 \pm 0.08$ & $11.45 \pm 0.02$ \\
\hline Au & M & $1.16 \pm 0.04$ & $0.20 \pm 0.01$ \\
\hline Total & & 100.00 & 100.00 \\
\hline \multicolumn{3}{|l|}{ Spc_o03 } & tting ratio 0.0073 \\
\hline
\end{tabular}




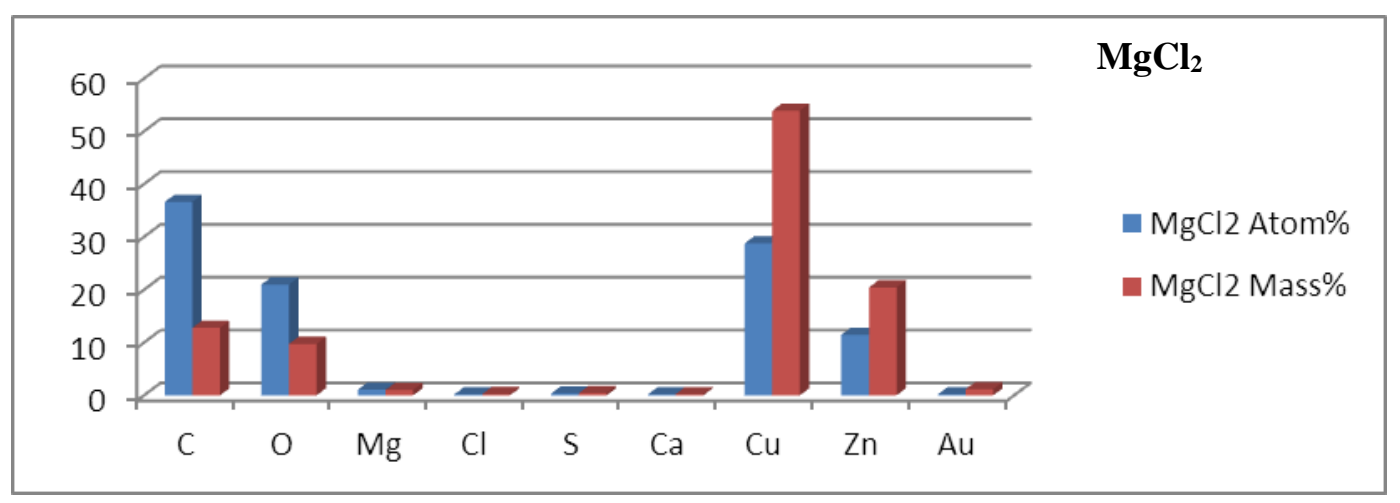

Figure 18. Graph comparing the elements in bronze sample affected by $\mathrm{MgCl}_{2}$ salt

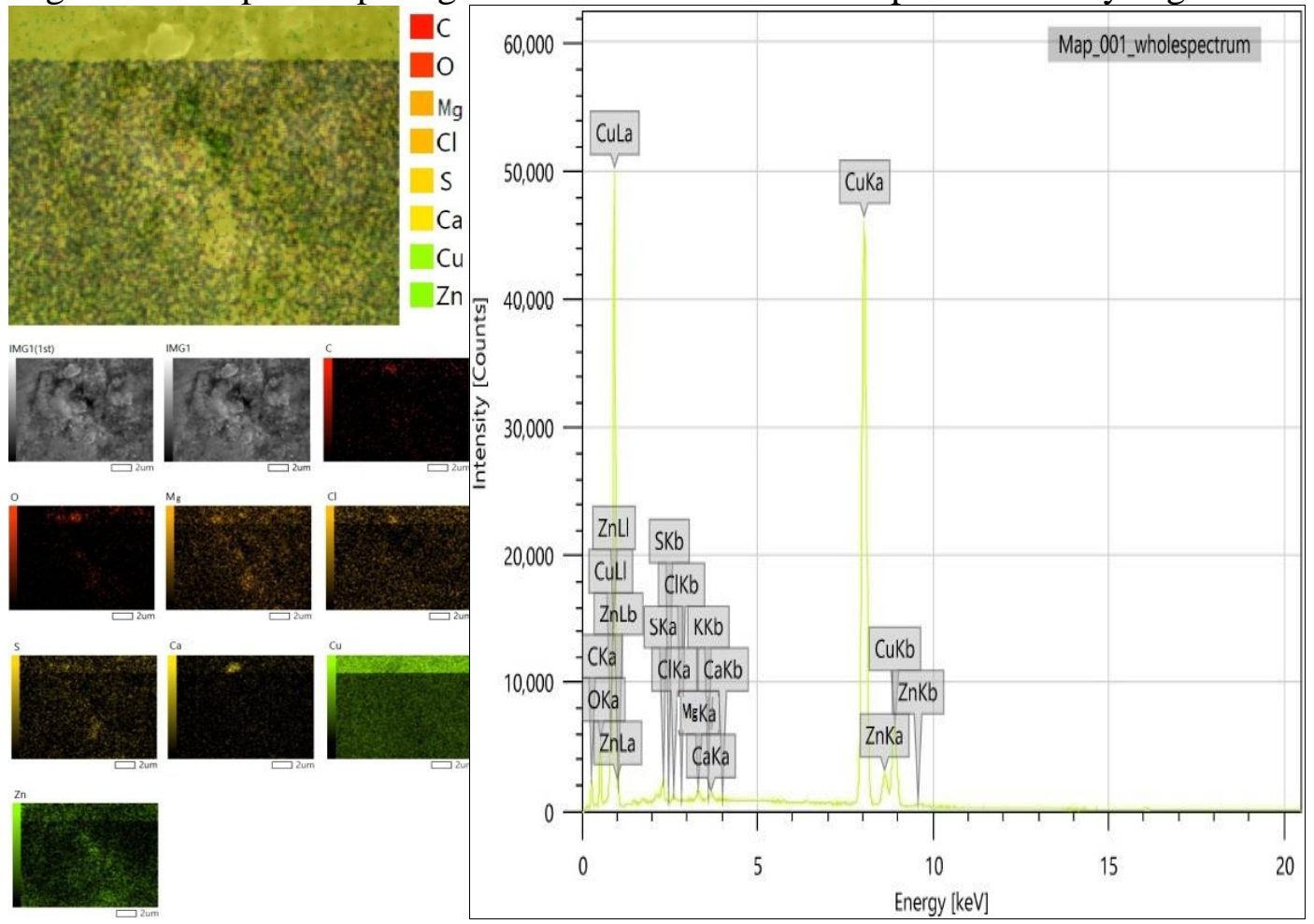

Figure 19. Mapping with chart show the spread of elements in the sample by coloring each element with a deferent color in bronze sample affected by $\mathrm{MgCl}_{2}$ salt

In sample No.(3) Sodium Bromide $(\mathrm{NaBr})$, the result of analyze showed elements of copper $(\mathrm{Cu})$ with percent $36.80 \%$ and Zinc (Zn) with percent $19.07 \%$ as a main component of bronze alloy. The elements of Carbon (C), and Oxygen (O) with percent $14.68 \%, 20.61 \%$ respectively. Then the Sodium $(\mathrm{Na})$ element with percent $6.06 \%$, and Brome $(\mathrm{Br})$ element with percent $2.65 \%$, and Chloride $(\mathrm{Cl})$ element with percent $0.13 \%$ as a trace (Figure 20, 21) (Table 6). And the mapping with chart also showed the spread of elements in the sample (Figure 22).

So we can to conclude to the Sodium Bromide salt has a bad effect on the bronze alloy this is due to the low copper percent, which means that there is corrosion in the sample with high oxygen percent. And appearance of chloride element may be as an air impurity. 


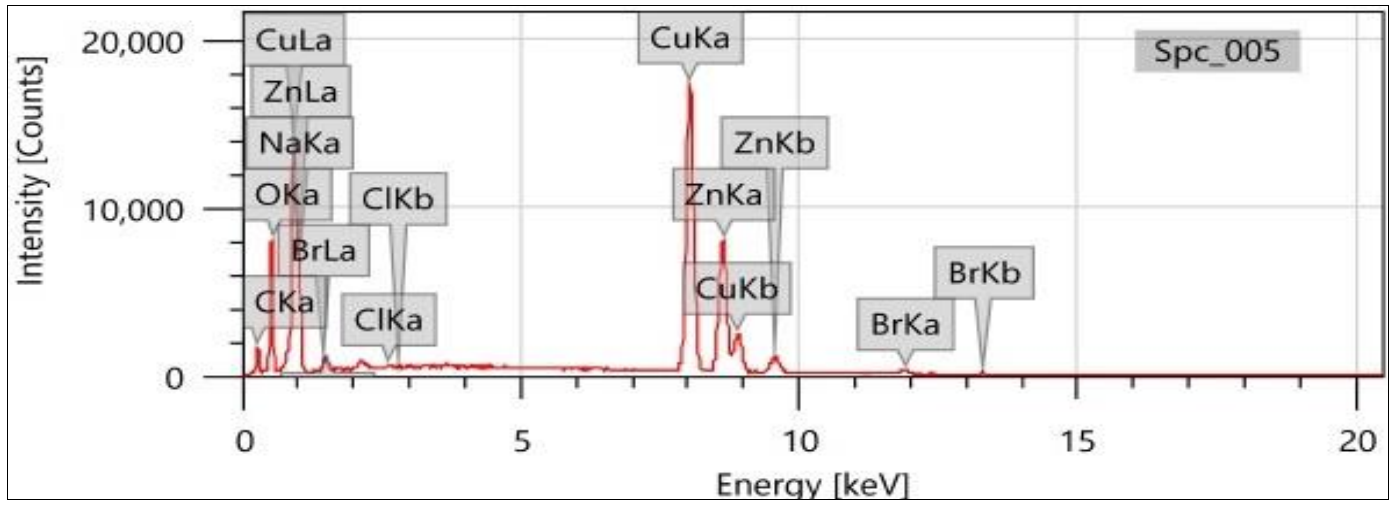

Figure 20. EDS analyses evident the elements in bronze sample affected by $\mathrm{NaBr}$ salt

Table 6. Percents of mass and atom of each element in bronze sample affected by $\mathrm{NaBr}$ salt

\begin{tabular}{|c|c|c|c|}
\hline Display name & \multicolumn{1}{|c|}{ Standard data } & Quantification method & Result Type \\
\hline Spc_005 & Standardless & ZAF & Metal \\
\hline
\end{tabular}

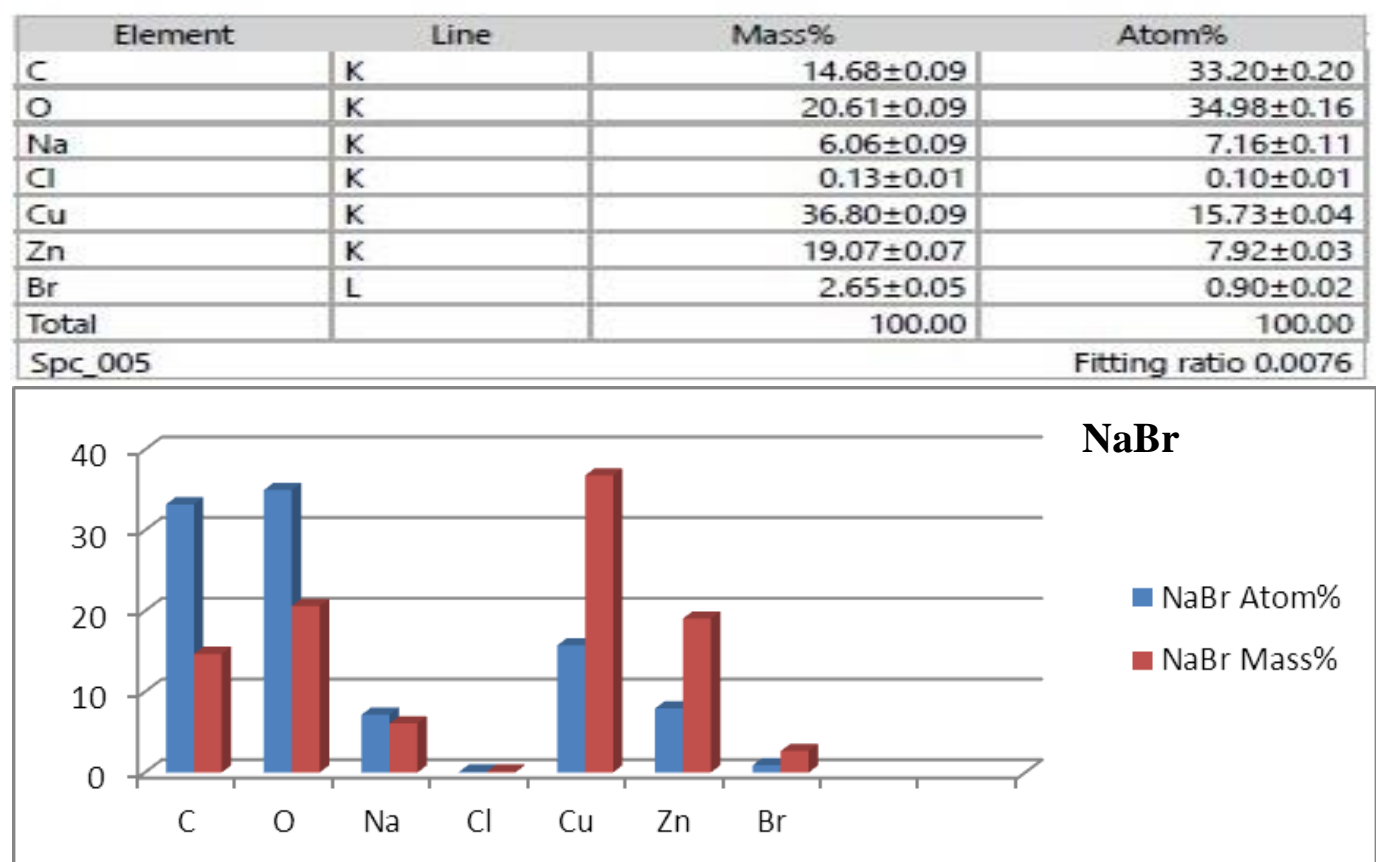

Figure 21. Graph comparing the elements in bronze sample affected by $\mathrm{NaBr}$ salt 

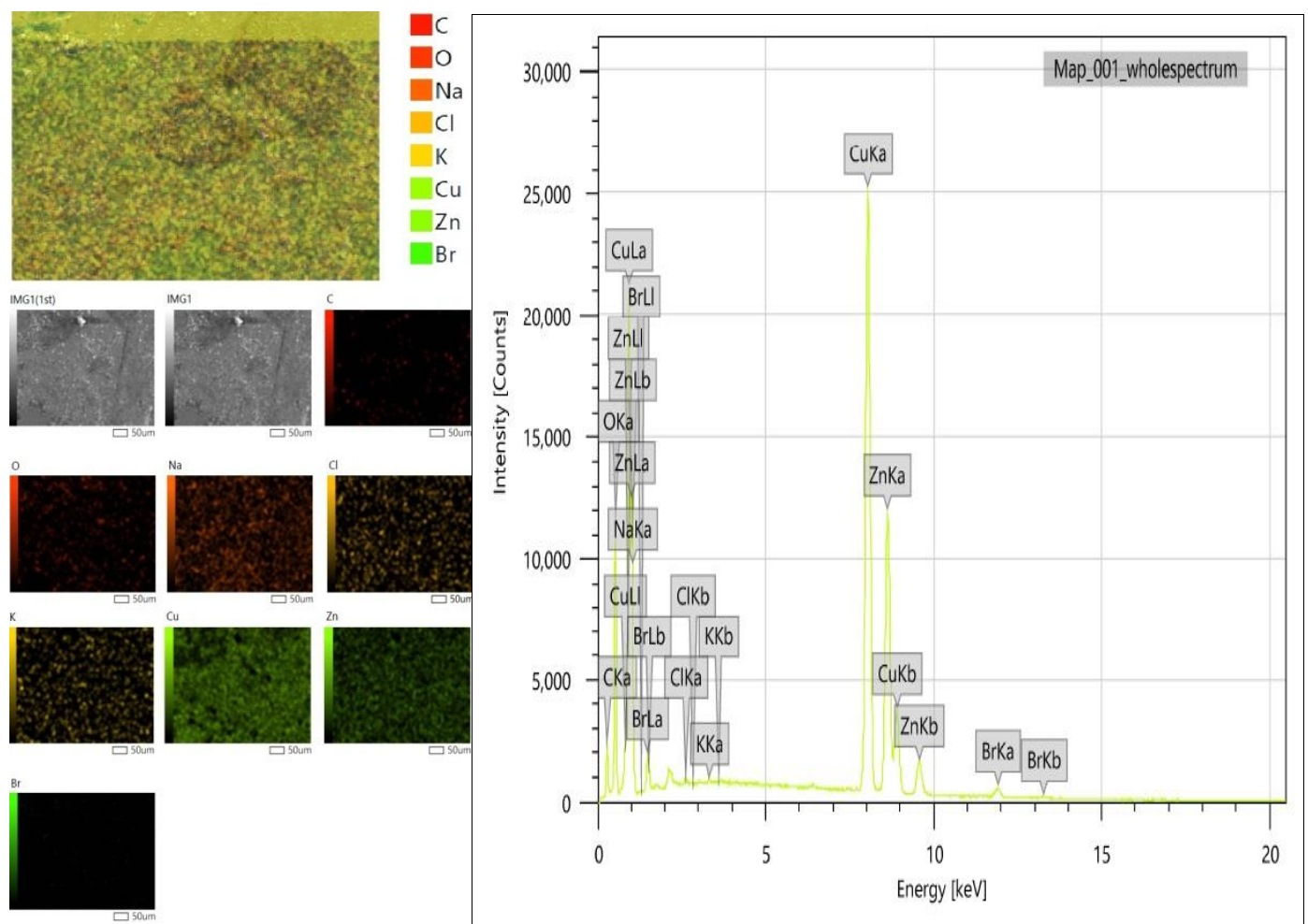

Figure 22. Mapping with chart show the spread of elements in the sample by coloring each element with a deferent color in bronze sample affected by $\mathrm{NaBr}$ salt

5.

CONCLUSION

All previous results were proved by digital microscope, SEM-EDS, SEM-EDX and Tensile Strength and Elongation; since the results revealed that Potassium carbonate salt can be safely used as a saturated salt solution for the museum relative humidity control with bronze metals. It didn't affect to the mass of the bronze sample. It can be use with textiles for two weeks. The results revealed that Magnesium chloride is not recommended for use with bronze metals because it was decreased the copper mass as a basic composite element in the bronze alloy due to the formation of rust compounds. It can be safely used with textiles because it was gave a suitable range of RH measurements and wasn't deposited on the samples. The results revealed also that Sodium bromide is not recommended for use with bronze metals because it has a bad effect on the bronze alloy this is due to the low copper percent which means that there is corrosion in the sample. It is also not suitable for use with textile because it was gave unsuitable range of RH measurements and deposited on the samples.

6.

REFERENCES

1-Agrawal O. P., 1987. Conservation of Metals-Problems and Prospects. Proceedings of the Asian Regional Seminar. In: Conservation of Metals in Humid Climate. (eds): O. P. Agrawal, ICCROM, NRLC, (Rome), 1.

2-Ahmed H. E., 2013. Proteaes Enzyme Used For Artificial Ageing on Modern Cotton Fabric for Historic Textile Preservation and Restoration. International Journal of Conservation Science, 4, 2, ISSN: 2067-533X, 177-188. 
3-Bacci M., Cucci C., Azelio Mencaglia A. A., Mignani A. G., 2005. Innovative Sensors for Environmental Monitoring in Museums", Sensors 8, 1984-2005.

4-Blaine R. , 2004. Humidity Fixed Points. TA Instruments, USA, 1-3.

www.tainstruments.com/pdfliterature/TN056.pdf

5-Brown S., Cole I., Daniel V., King S., Pearson C.,2001. Guidelines for Environmental Control in Cultural Institutions - Consortium for Heritage Collections and their Environment. Heritage Collections Council, Collections Development Section, Department of Communications, Information Technology and the Arts, Australia, 10-11.

6-Cassar M., and Hutching J., 2000. Relative Humidity and Temperature Pattern Book. The Museums \& Galleries Commission (MGC), London, 2000, 4.

7-Dostal J., 2013. Climatic Unit Control. Diploma Thesis, Czech Technical University in Prague, Faculty of Electrical Engineering, Department of Control Engineering, Climatic Unit Control, (Prague), 53-54.

8-Elgaoudi H., Kourkoumelis N., Varella E., Kovala-Demertzi D., 2011. The effect of thermal aging and color pigments on the Egyptian linen properties evaluated by physicochemical methods. Applied Physics A, 105, 497-507.

9-Feller R. L., 1994. Accelerated aging, photochemical and thermal aspects. USA, The Getty Conservation Institute, 161.

10-Forney C. F.and Brandl D. G. , 1992. Control of Humidity in Small Controlled environment Chambers using Glycerol-Water Solutions. Hort Technology 2 , 1, 52-54.

11-Georgianna M., Polytimi L., Panagiotis S., 2007. Azoton ${ }^{\mathrm{TM}}$, a Nitrogen climatic cabinetfor the trans-portand safe storage of organic archaeological finds. In: Abstracts of posters at the Copenhagen conference, 19 - 23 November, Museum Microclimates, (eds): T. Padfield and K. Borchersen, The National Museum of Denmark, 10.

12-Greenspan L., 1977. Humidity Fixed Points of Binary Saturated Aqueous Solutions. Journal of Research of the National Bureau of Standards - A. Physics and Chemistry 81 A, 1, 89-96.

13-Henderson J., 2007. Managing the library and archive environment. Preservation Advisory Centre, The British Library, London, 4-5.

14-Hyland R. W., C. and Hurley W., 1983. General Guidelines for the On-Site Calibration of Humidity and Moisture Control Systems in Buildings. U.S. Department of Commerce, National Bureau of Standads, U.S. Government Printing Office, Washington DC 20234, 10.

15-Jialu C., 2011. Broadband Dielectric Properties of Impregnated Transformer Paper Insulation at Various Moisture Contents. Master's Degree Project, Electromagnetic Engineering, School of Electrical Engineering, Kungliga Tekniska Hogskolan, Stockholm, Sweden, 56-57.

16-Jowitt R. and Wagstaffe P. J., 1989. The Certification of the Water Content of Microcrystalline Cellulose (MCC) at 10 Water Activities. Commission of the European Communities, EUR 12429 EN, Luxembourg, 10.

17-Lampropoulos V., Kontonikoli M., Papadimitriou M., Karampotsos, A., 2007. Preservation conditions of roman glass vessels of 1 century ad from Rhodes Island - Greece. In: Abstracts of posters at the Copenhagen conference, 19 - 23 November, Museum Microclimates, (eds): T. Padfield and K. Borchersen, The National Museum of Denmark, 19.

18-Quincot G., Azenha M., Barros J., Faria R., 2011. Use of salt solutions for assuring constant relative humidity conditions in contained environments. Projetos De Investigacao Cientifica E 
Desenvolvimento Tecnologico, União Europeia - Fundos Estruturais, Governo da República Portuguesa, Guimarães, UM , 1-3.

19-Rimmer M., and Wang Q., 2010. Assessing the effects of alkaline desalination treatments for archaeological iron using scanning electron microscopy. The British museum, Technical Research Bulletin 4, 79.

20-Romano F., Gaudenzi M., Joppolo C.M. Romano L. P., 2013. Temperature and humidity in museum display case: a simulation tool and experimental validation. Built Heritage, Monitoring Conservation Management, 1329-1335.

21-Slade S., 2003. Temperature and Humidity. Museums Australia Victoria, Scottish Museums Council Fact Sheet - adapted for use in Australia, 1.

22-Trustees of the Royal Air Force Museum, RAFM 2008. The Royal Air Force Museum's Policy for Monitoring Relative Humidity and Temperature. Environmental Monitoring Policy - RH and Temperature, London, RAFM/DCM/2/6/25/1, 1-3.

23-Wexler A., and Hasegawa S., 1954. Relative Humidity-Temperature Relationships of Some Saturated Salt Solutions in the Temperature Range 0 to 50 C. Journal of Research of the National Bureau of Standards 53, 1, 19-26. 